\title{
Noise reduction in astronomical spectra using wavelet packets
}

\author{
M. Fligge and S.K. Solanki \\ Institut für Astronomie, ETH-Zentrum, CH-8092 Zürich, Switzerland
}

Received May 15; accepted December 6, 1996

\begin{abstract}
The wavelet representation of a signal offers greater flexibility in de-noising astronomical spectra than classical Fourier smoothing due to the additional wavelength resolution of the decomposed signal. We present here a new wavelet-based approach to noise reduction. It is similar to an application of the splitting algorithm of a wavelet packets analysis using non-orthogonal wavelets. It clearly separates the signal from the noise, in particular also at the noise-dominated highest frequencies. This allows a better suppression of the noise, so that the spectrum de-noised in this manner provides a closer approximation of the uncorrupted signal than in the case of a single wavelet transformation or a Fourier transform.

We test this method on intensity and circularly polarized spectra of the sun and compare with Fourier and other wavelet-based de-noising algorithms. Our technique is found to give better results than any other tested denoising algorithm. It is shown to be particularly successful in recovering weak signals that are practically drowned by the noise.
\end{abstract}

Key words: methods: data analysis - methods: numerical

\section{Introduction}

Astronomical observations are often photon starved. Consequently many astronomical spectra have a poor signal-to-noise ratio (SNR) and are significantly corrupted by (white Gaussian) photon noise. This is even true for solar observations, since high resolution measurements of polarized light soon run out of photons (e.g., Stix 1991). The reduction of this noise is highly desirable for a number of reasons (cf. the papers in the volume edited by Cayrel De Strobel \& Spite 1988, for examples of the merits of high SNR spectra).

Send offprint requests to: M. Fligge
Fourier smoothing has long been the method of choice to suppress noise (Brault \& White 1971), but recently methods based on the wavelet transformation have become increasingly popular (Starck \& Bijaoui 1994; Starck \& Murtagh 1994; Murtagh et al. 1995). In principle they offer much greater flexibility for analyzing and processing data. The main advantage of wavelets lies in the additional "spatial" resolution of the transformed signal. In contrast to the Fourier transformation, the signal is decomposed into waves of finite length, i.e. into waves which are spatially localized - hence the name wavelets. The wavelet transform of a one-dimensional signal has two independent variables - a frequency and a spatial location variable. It leads to a decomposition of, say, a spectrum into a series of spectra at finer and coarser resolutions. Indeed, there is a close mathematical relationship between the wavelet transformation and the multi-resolution analysis of a signal (Mallat 1989). Hence the wavelet transform furnishes us with the frequency spectrum of a signal at every spatial location. This feature, besides others, opens new and fruitful ways of processing and analyzing data of various kinds.

In particular, it allows the smoothing of astronomical spectra, typically composed of a continuum with interspersed spectral lines, to be optimized. Since highfrequency signals are present only at the wavelengths of the spectral lines, only at these wavelengths need they be kept in the de-noised spectrum. At the remaining positions, i.e. in the continuum, only the lowest frequencies are due to the source itself. Whereas Fourier filtering affects all data points in the same manner, wavelets allow different parts of spectra to be filtered individually, in principle promising a considerably refined and improved treatment.

In the present paper, we compare different wavelet smoothing methods to each other and to Fourier smoothing for the specific case of astronomical spectra. We also present a wavelet-packets based smoothing scheme which we find to be superior in recovering the true signal from a combination of signal and noise, at least for the cases we have considered. 


\section{Some relevant properties of wavelets}

The Fourier transformation decomposes a signal into sines and cosines of different frequencies. The wavelet transformation acts similarly, but instead of non-local, strictly periodic sines and cosines, it uses a set of spatially localized functions $\psi_{a, b}(x)$ called wavelets (Daubechies 1988; Meyer 1993; see Press et al. 1992 for a simple introduction to the subject). The wavelets are constructed by translating and dilating a mother wavelet $\psi(x)$

$\psi_{a, b}(x)=\frac{1}{\sqrt{|a|}} \psi\left(\frac{x-b}{a}\right) \quad(a \neq 0)$,

where the scale parameter $a$ plays the role of a frequency and $b$ is the position parameter. By increasing $a$, the wavelet $\psi_{a, b}(x)$ is broadened, while changing $b$ translates it along the $x$-axis. The set of parameters $(a, b)$ describes a point in the so called scale-space plane.

The continuous wavelet transform of a function $f(x)$, $W_{\mathrm{c}} f(a, b)$, is defined by

$W_{\mathrm{c}} f(a, b)=\left\langle\psi_{a, b}(x), f(x)\right\rangle=\int_{-\infty}^{\infty} f(x) \psi_{a, b}^{*}(x) \mathrm{d} x$.

It is invertible (Grossmann \& Morlet 1984) and the function $f(x)$ can be recovered by evaluating the double integral:

$f(t)=\frac{1}{\Lambda} \int_{-\infty}^{\infty} \int_{-\infty}^{\infty} W_{\mathrm{c}} f(a, b) \psi_{a, b}(x) \frac{\mathrm{d} a \mathrm{~d} b}{a^{2}}$.

Note that, unlike the Fourier transform, the wavelet transform is not its own inverse. This implies that a signal may be transformed several times using wavelets and be further decomposed at each transformation. Such a sheme of repeated application of the wavelet transform leads to the splitting algorithm of a wavelet packets analysis (Wickerhauser 1991, 1994; Chui 1992b) which lies at the heart of the technique we propose.

For practical applications, the continuous set of parameters $(a, b)$, must be discretized (Daubechies 1988; Mallat 1989). The parameterization of the discrete $(a, b)$ pairs is of crucial significance to the discrete wavelet transform and especially to the stability of the reconstruction algorithm (Daubechies 1990). For most of the parameterizations of $(a, b)$ the set of $\left\{\psi_{a, b}(x)\right\}$ is highly redundant, i.e. each subset of them can be generated by linear combinations of the others (Daubechies et al. 1986). Although in such cases the reconstruction is not exact anymore, such a decomposition has a remarkable advantage when considering de-noising applications (Daubechies 1990).

The particular class of non-orthogonal 1-D wavelets and the corresponding discrete wavelet transform together with the numerical algorithm we have used was proposed by Mallat \& Zhong (1992, see their Appendix A). The multi-level decomposition described in Sect. 3.2 is a direct application of wavelet-packets using this particular kind of base functions.

\section{Methods}

\subsection{Filtering and de-noising methods based on wavelets}

Most of the wavelet coefficients of the transform of a noiseless signal are close to zero. Therefore the most obvious way of filtering in the wavelet domain is to identify those wavelet coefficients that are significantly non-zero against the noisy background (Donoho \& Johnstone 1994). This motivates the method of hard thresholding according to which only the significantly non-zero coefficients are retained and all others rejected:

$\begin{array}{ll}W_{m}=0 & \text { if }\left|w_{m}\right|<k \sigma_{m} \\ W_{m}=w_{m} & \text { if } \quad\left|w_{m}\right| \geq k \sigma_{m}\end{array}$

where the $w_{m}$ are wavelet coefficients of the noisy signal, the $W_{m}$ are the filtered wavelet coefficients, $\sigma_{m}$ is the estimated standard deviation of the noise at the appropriate scale $m$, and $k$ is a freely selectable factor which defines the level of confidence for the preserved coefficients. Non-orthogonal wavelets reduce the $\sigma_{m}$ with increasing $m$, and hence we have to estimate it for each scale separately (Starck \& Bijaoui 1994).

We now briefly introduce the principles of some more sophisticated de-noising methods with which we later compare our own method. The first method was developed by Donoho $(1992 \mathrm{a}, \mathrm{b})$. It is based on the so-called wavelet shrinkage technique. In addition to the application of a threshold criterion, the wavelet coefficients are shrunk towards zero. Donoho \& Johnstone (1992, 1993, 1994) provide several algorithms based on statistical arguments to determine the level of shrinkage.

Another set of noise-reduction methods have been proposed by Starck \& Bijaoui (1994). They employ the socalled $\grave{a}$ trous algorithm to perform the wavelet transformation based on linear or cubic spline functions. They apply different filter criteria which include information on wavelet coefficients of different scales (hierarchical filtering). In addition, they use an iterative reconstruction (adaptive filtering) to find a signal whose wavelet transform is as close as possible to the original set of thresholded coefficients (see also Starck \& Murtagh 1994 and Starck et al. 1995 for further details).

The final noise reducing technique to which we compare our method was developed by Bury et al. (1996) and is also based on the $\grave{a}$ trous algorithm. They apply an iterative structure detection algorithm in the scale-space plane to extract the significant coefficients from the noisy wavelet transform.

In the next section we present a somewhat different approach to the problem of noise reduction using waveletbased techniques. Instead of applying a suitable selection criterion to determining the significant coefficients of a certain scale we extract the underlying spectral features of a noisy scale by simply transforming it again using the same wavelet base (wavelet-packets analysis). The transformed 

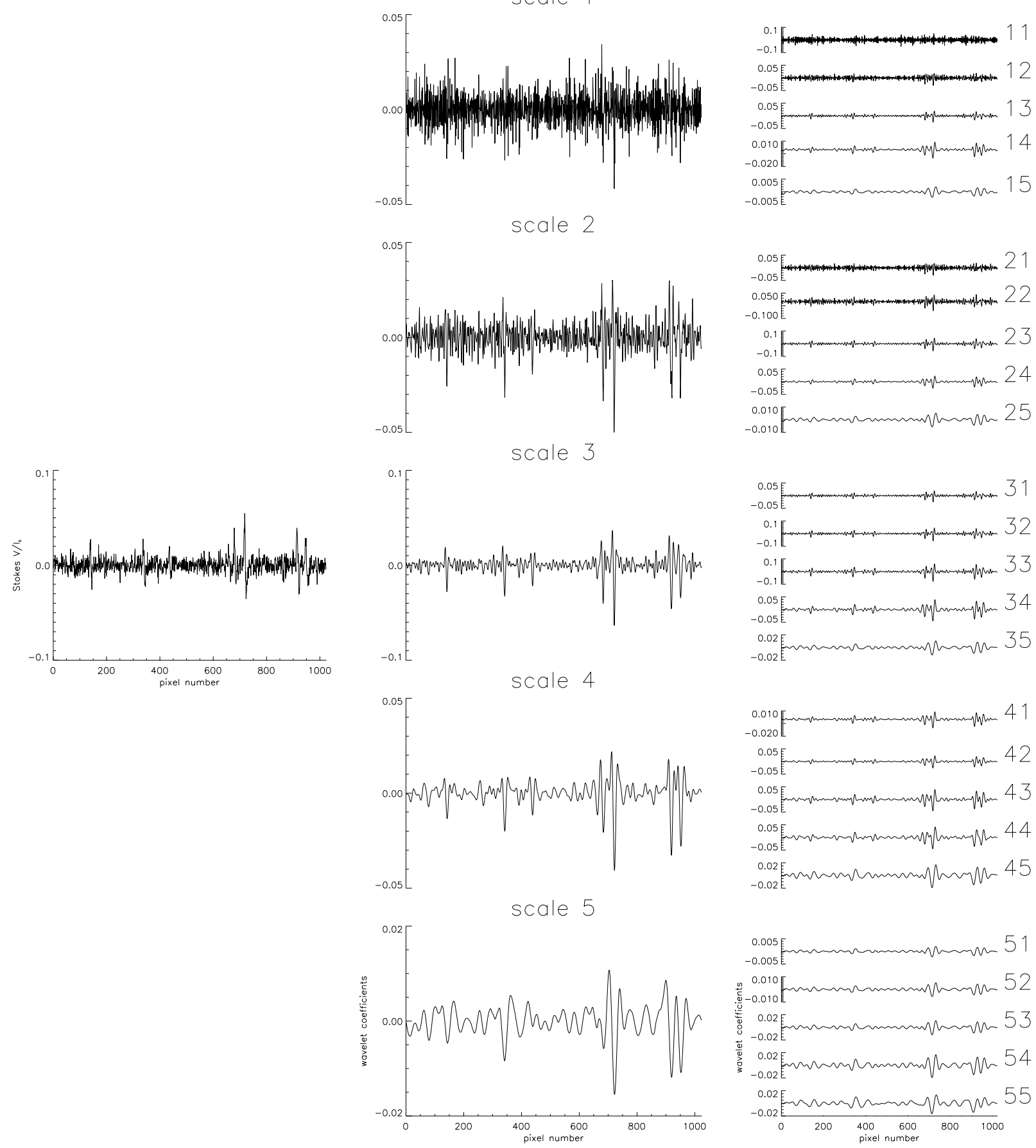

Fig. 1. Two-level decomposition of a Stokes $V$ spectrum with a noise level of $\sigma_{\text {noise }}=0.0056$. The corrupted signal is plotted on the left, the coefficients of the first level of decomposition are plotted in the central column. The coefficients of the wavelet transform of each of the scales in the central column are plotted on the right (second level of decomposition). The two-level decomposition reveals some of the uncorrupted signal at scales 13 to 15 which is absent at scale 1 . Hence truncating scales 11 to 15 , instead of scale 1 , can recover features of the uncorrupted signal which are lost if scale 1 is truncated 
signal is then de-noised by a simple hard thresholding criterion. Details are given in the next section.

\subsection{De-noising using wavelet-packets}

Noise is mainly concentrated at the finest scales of a signal transformed using non-orthogonal wavelets, i.e. the signalto-noise ratio (SNR) decreases towards the finer scales. If the noise level of the original signal is sufficiently high, the finest scales are completely dominated by noise. As an example consider the noisy signal in the left part of Fig. 1 (details about the signal are given later). The wavelet coefficients of the noisy signal are plotted at the center, with frequency decreasing from top to bottom. The highest frequency coefficients, i.e. the top row, are completely dominated by noise. As we demonstrate later, however, there is a considerable signal hidden in that noise. Simply truncating the wavelet coefficients at these scales either rejects the real signal along with the noise or leaves so much noise that the signal is still not recognizable. This dilemma is resolved using the splitting algorithm of a wavelet-packets analysis (Wickerhauser 1991). Each scale (frequency) may itself be considered to be a noisy signal for which we wish to separate the noise from the signal. We do this by transforming (decomposing) the wavelet coefficients at each scale, again, exactly in the same manner as we transformed the original signal. The coefficients resulting from this second set of transformations are shown on the right side of Fig. 1: each scale of the singly transformed signal gives rise to (in this case) 5 scales in the doubly transformed or twice decomposed signal.

For greater clarity we number the scales of the different decompositions as follows: The scales after the first transformation are numbered $1,2, \ldots 5$ (from finest to coarsest). After the second level of decomposition we number them as follows: $11,12, \ldots 15$ (transforms of scale 1 ), ..., 51, 52, ... 55 (transforms of scale 5). The tree-like arrangement of the individual scales which results from a two-level decomposition of a Stokes $V$ (i.e. circularly polarized) spectrum is illustrated in Fig. 1.

Consider scale 1 of Fig. 1. It is completely dominated by noise. Simple thresholding will set each coefficient to zero, because none lies significantly above the background noise. Consequently the restored signal will be considerably altered in the high frequency range, i.e. in the peaks of the spectral lines. After the second transformation the hidden signal has partially been separated from the noise and has become clearly visible at the scales 14 and 15 and can still be detected in scale 13, although just barely. If we now truncate all the wavelet coefficients of the twice decomposed signal under a hard threshold, then we can reduce the noise by almost the same amount as after the first transformation, while preserving much more of the uncorrupted signal, particularly at high frequencies. It is possible to recover even more of the original signal by transforming scales 11-55 once more (three-level decom-
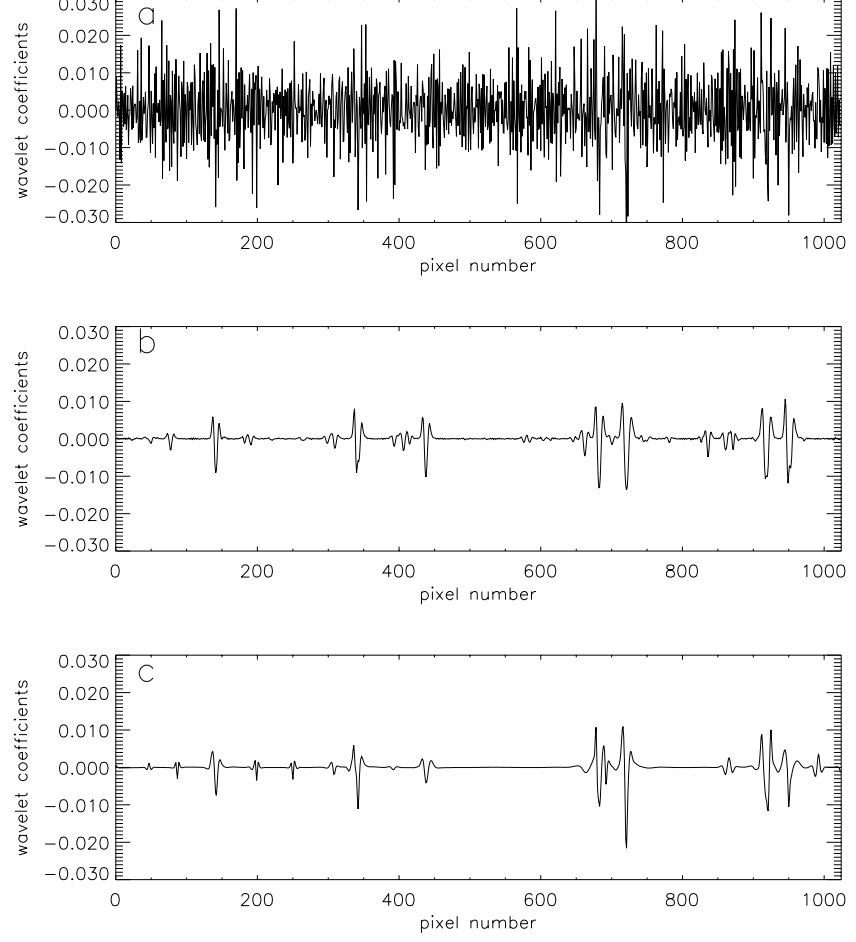

Fig. 2. a) Finest scale of the non-orthogonal wavelet transform of a noisy signal (scale 1 of Fig. 1). b) Same scale of the uncorrupted original signal. c) Reconstructed scale after a three-level-decomposition. Clearly there is little chance of recovering the uncorrupted pattern out of the noisy signal a) by simply truncating at a certain threshold value. In contrast the reconstructed scale c) is a good approximation of the uncorrupted scale b)

position). The degree to which a three-level decomposition can restore scale 1 is shown in Fig. 2. Note the remarkable similarity of the reconstructed coefficients (Fig. 2c) to the original, uncorrupted coefficients (Fig. 2b).

Due to its high redundancy the base of non-orthogonal wavelets has the highly desirable property that it allows the main features of the uncorrupted signal to be recognized even after several decompositions of the noisiest scales. So instead of truncating the coefficients of the finest scales directly it is worth transforming them once again and performing the truncation on the coefficients of the second (or third) decomposition level.

Generally, by truncating the coefficients of a higher level of decomposition, it is possible to restore features of the uncorrupted signal at scales of lower levels, which were originally drowned in the noise. This is especially effective for the finest scales whereas the coarser scales have a larger SNR and hence can be truncated directly at the first level of decomposition without affecting their information content significantly.

In the present paper we truncate the coefficients on the basis of a simple hard thresholding criterion. Since we are dealing with non-orthogonal wavelets, we need to know the standard deviation of noise at each scale $\sigma_{\text {noise, } m \text {. Due }}$ 
to the linearity of the wavelet transformation, i.e.

$$
\begin{aligned}
W(f+g)(a, b) & =<\psi_{a, b}(x), f(x)+g(x)> \\
& =<\psi_{a, b}(x), f(x)>+<\psi_{a, b}(x), g(x)> \\
& =W f(a, b)+W g(a, b),
\end{aligned}
$$

this can be obtained from the transformation of a signal that consists purely of noise. Strictly speaking we do the following. First we determine the standard deviation of the noise of the corrupted signal (white Gaussian noise in the illustrated case). Then this value is used to create a signal of pure, e.g., white Gaussian noise to which our splitting scheme is applied. Finally, we get an estimate of the standard deviation of noise at scale $m$ of the corrupted signal, called $\sigma_{m}$, by calculating the standard deviation of the pure noise, called $\sigma_{\text {noise, } m}$, of that scale. Such a scheme has already been applied by Starck \& Bijaoui (1994). We set the threshold value to $k \cdot \sigma_{m}$. The factor $k$ determines the confidence level of the preserved coefficients. In general we set $k=3$, which results in a level of confidence of $99.7 \%$.

\subsection{Data and noise model}

We test the various methods on intensity (Stokes $I$ ) and net circular polarization spectra (Stokes $V$ ) of solar features recorded with the Fourier Transform Spectrometer (FTS) installed at the McMath Pierce facility at Kitt Peak. The data are ideal for testing various de-noising techniques for a number of reasons. They have a very low noise level (as low as $10^{-4} I_{\mathrm{c}}$ in some parts of the spectrum; $I_{\mathrm{c}}$ is the continuum intensity), so that de-noising techniques can be tested rigorously. They have a spectral resolving power of $360000-500000$, so that the high frequencies present in the original solar spectrum have not been significantly suppressed by the instrument. They cover a broad wavelength range with different types of spectral features. Also, both Stokes $I$ and Stokes $V$ spectra were recorded. In the following we first concentrate on Stokes $V$ since it shows higher frequencies and thus poses a larger challenge to de-noising techniques, particularly since the data are sampled close to the Nyquist frequency. The data are described in detail by Stenflo et al. (1984) and Solanki (1987).

We first corrupted the spectra by adding artificial white Gaussian noise whose standard deviation scales as the inverse of the square root of the reference spectrum intensity. Such a noise spectrum is a good representation of Poisson distributed photon noise in the limit of many photons (which is fulfilled by the present data) ${ }^{1}$. These noisy spectra then served as input data for the de-noising algorithms described in Sects. 3.1 and 3.2.

1 We also considered somewhat deviant noise distributions which gave only insignificantly different results and will not be discussed further.
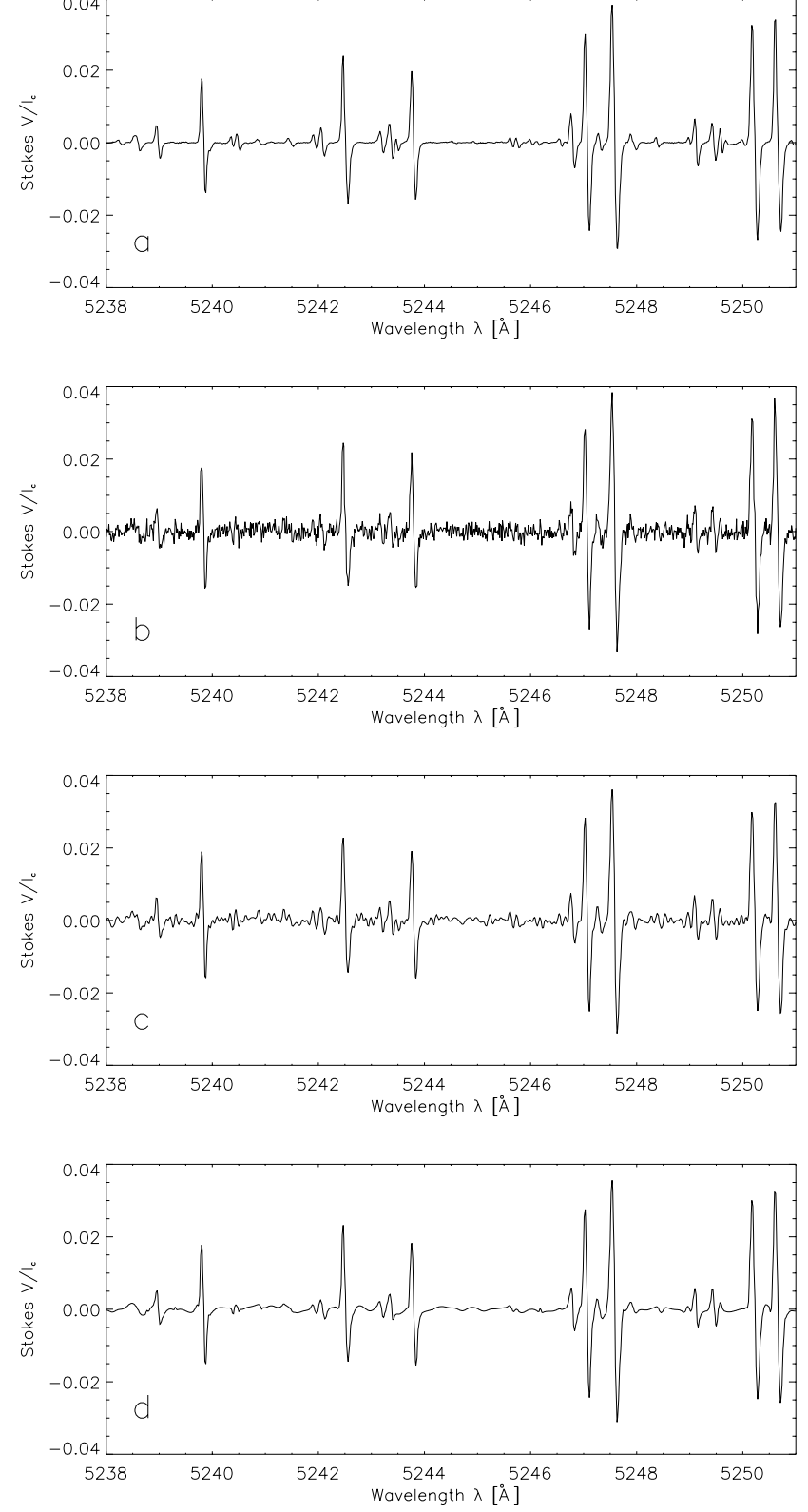

Fig. 3. a) Uncorrupted Stokes $V$ spectrum. b) Corrupted version with artificially added noise with $\sigma_{\text {noise }}=0.0014$. c) Fourier smoothed spectrum. It shows clearly the typical random oscillations within the low frequency parts of the spectrum. d) Wavelet-packets de-noised version, using a three-level decomposition. The continuum is now clean and even a number of small features of the original spectrum are at least qualitatively recovered

\section{Results}

We compare the quality of different wavelet-based denoising techniques presented in Sects. 3.1 and 3.2 by applying them to originally low-noise solar spectra that have been artificially contaminated by noise as described in Sect. 3.3. We also compare these techniques with the Fourier filter giving the best results, as found by trial and error. In general the original, uncorrupted spectrum is not 
known a priori and the goodness of the Fourier smoothing will be lower than the value we obtain.

The added noise has a standard deviation of $\sigma_{\mathrm{c}}=$ 0.0014 in the continuum of the Stokes $V$ signal and a $\sigma_{\mathrm{c}}=0.014$ for the Stokes $I$ spectrum. We first de-noise a solar Stokes $V$ spectrum. Later we illustrate the techniques further by applying them to a Stokes $I$ spectrum.
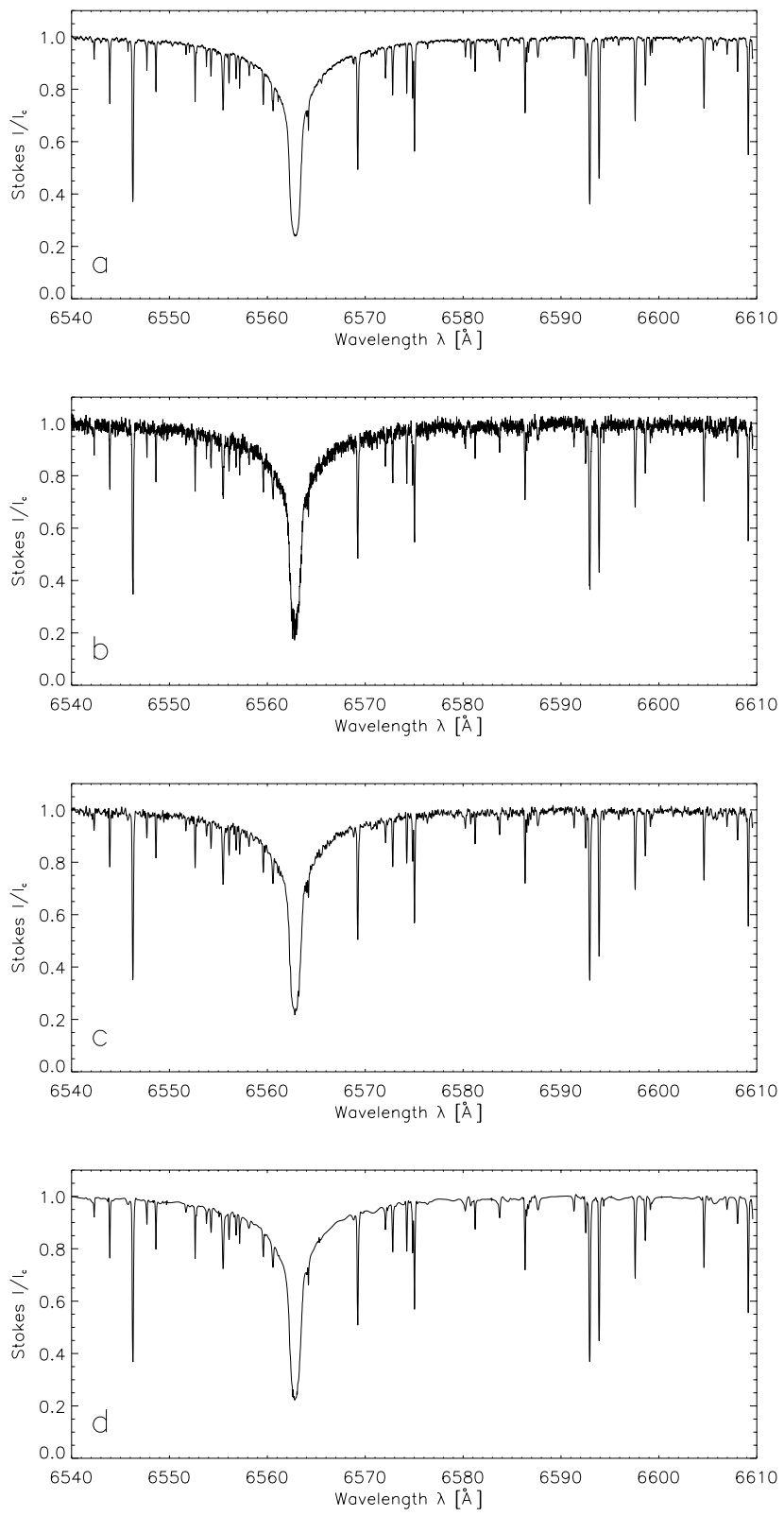

Fig. 4. a) Uncorrupted Stokes $I$ spectrum. b) Corrupted version $\left(\sigma_{\text {noise }}=0.014\right)$. d) Fourier smoothed spectrum. d) Wavelet-packets de-noised spectrum using a three-level decomposition
We express the goodness of a de-noised spectrum by the variance $\sigma^{2}$,

$\sigma^{2}=\frac{1}{N-1} \sum_{j=1}^{N}\left(x_{j}-\overline{x_{j}}\right)^{2}$,

where $\left\{x_{j}\right\}, j=1 \ldots N$, represents the uncorrupted signal and $\left\{\overline{x_{j}}\right\}, j=1 \ldots N$ stands for the de-noised version of the signal. $N$ is the number of spectral points. Therefore the standard deviation of the remaining noise of a denoised version of a spectrum is simply the square root of the variance.

To obtain a clearer picture of what the de-noising algorithm does in detail we evaluate different types of spectral features individually. In addition to the whole spectral range we consider separately the strong lines (four strongest features between $5247 \AA$ and $5251 \AA$ ), the weak lines (four strongest features between $5239 \AA$ and $5247 \AA$ ) and the continuum regions, respectively. Note that the "continuum" includes the (roughly 20) very weak spectral features visible in Fig. 3a. Since the spectral lines and in particular the amplitudes of their peaks are of special interest in astronomical spectral analysis, the threshold value for the Fourier method is adapted to the spectral lines, i.e. it is chosen to restore them as well as possible (therefore the success of the Fourier smoothing for the weak lines must be seen in this context). Since Fourier smoothing cannot remove noise at all frequencies equally well it consequently fails within the continuum. As a result, very small features, although still present in the spectrum, are hardly recognizable, because they are sometimes completely embedded in the random oscillations typical for Fourier smoothed signals. If the Fourier low-pass threshold is lowered a clearer continuum results, but the spectral lines are distorted due to missing high frequencies.

In contrast, our wavelet-packets technique is able to de-noise both the high frequency (spectral lines) and low frequency (continuum) features simultaneously (Fig. 3). Table 1 confirms this. The ratio $\sigma_{\text {noise }} / \sigma$ is a measure of the reduction in the noise level over the whole signal due to the de-noising algorithm. The Fourier method reduces the noise of all spectral features by about the same amount, it halves $\sigma_{\text {noise }}^{2}$ everywhere, whereas the wavelet techniques reduces noise within the continuum much more strongly and hence clearly reveals even very small features of the uncorrupted spectrum which were completely swamped by the noise (Fig. 3). The exception are the wavelet shrinkage methods, whose results strongly depend on the used statistical threshold criterion. We were however not able to improve the result for the continuum regions without simultaneously increasing the distortion of the spectral lines.

Of the tested methods our wavelet-packets technique attains the best results. Due to the additional transformations, it is able to filter out the noisy coefficients better 
Table 1. Numerical evaluation of a de-noised Stokes $V$ spectrum

\begin{tabular}{lcclcl}
\hline & Fourier & $\begin{array}{c}\text { Wavelet } \\
\text { shrinkage } \\
\sigma^{2}\left[\cdot 10^{-7}\right]\end{array}$ & $\begin{array}{l}\text { Hierarchical } \\
\text { thresholding } \\
\sigma^{2}\left[\cdot 10^{-7}\right]\end{array}$ & $\begin{array}{l}\text { Structure } \\
\text { detection } \\
\sigma^{2}\left[\cdot 10^{-7}\right]\end{array}$ & $\begin{array}{l}\text { wavelet-packets } \\
\text { 3-level decomposition } \\
\sigma^{2}\left[\cdot 10^{-7}\right]\end{array}$ \\
\hline Whole spectrum & 9.4 & 9.4 & 7.1 & 7.5 & 6.5 \\
Strong lines & 19.2 & 20.0 & 20.0 & 20.0 & 17.2 \\
Weak lines & 7.0 & 8.8 & 8.7 & 8.1 & 8.3 \\
"Continuum" & 6.4 & 6.4 & 1.8 & 2.1 & 2.0 \\
\hline$\sigma_{\text {noise }} / \sigma$ & 1.55 & 1.55 & 1.78 & 1.73 & 1.86 \\
\hline
\end{tabular}

than every other tested de-noising algorithm, as may be seen by scrutinizing Table 1 . In the case of the Stokes $V$ spectrum of Fig. 3, the noise level of the continuum regions is reduced to $4.4710^{-4}$, which means 1.8 times better than the value obtained by Fourier smoothing. Averaged over the whole spectral range, the multi-level decomposition results in a gain of about $20 \%$ compared to the classical Fourier smoothing method.

The analysis of a Stokes $I$ spectrum shows the same basic results: see Fig. 4 and Table 2. This spectrum includes the strong $\mathrm{H} \alpha$ line and allows us to test the various techniques in the presence of a strong, heavily oversampled spectral line. Hence this spectrum was chosen, although it isn't quite ideal since the original spectrum already was contaminated by roughly $10^{-3} I_{\mathrm{c}}$ noise. Again, we artificially added noise with a standard deviation of $\sigma=0.014$ (continuum). Now the advantages of wavelet-based methods are even more pronounced than in the case of the Stokes $V$ spectrum. The wavelet techniques are able to significantly suppress the noise in all spectral regions and attain an increase of the SNR over the whole signal of about $10 \%$ to $30 \%$ relative to Fourier smoothing. Again, our non-orthogonal multi-level decomposition leads to a large gain in the low frequency parts of the spectrum relative to Fourier smoothing. A number of small features are clearly recognizable in the wavelet de-noised version of the signal whereas they are embedded in random oscillations in the case of Fourier smoothing (Fig. 4). Note that the wavelet-packets technique has also removed much of the noise present in the original spectrum (top row), which - due to its definition, Eq. (6) - results in a larger $\sigma$ in Table 2 than would be present if the original spectrum really were noise-free. Considering the whole spectral range, our wavelet packets technique again attains the best results and leads to a roughly $30 \%$ lower $\sigma_{\text {noise }}$ than the Fourier smoothing method.

To completely characterize the quality of the de-noised signal we employ a multi-resolution quality criterion as proposed by Starck \& Bijaoui (1994). Both the originally noise-free and the de-noised signal are compared in the
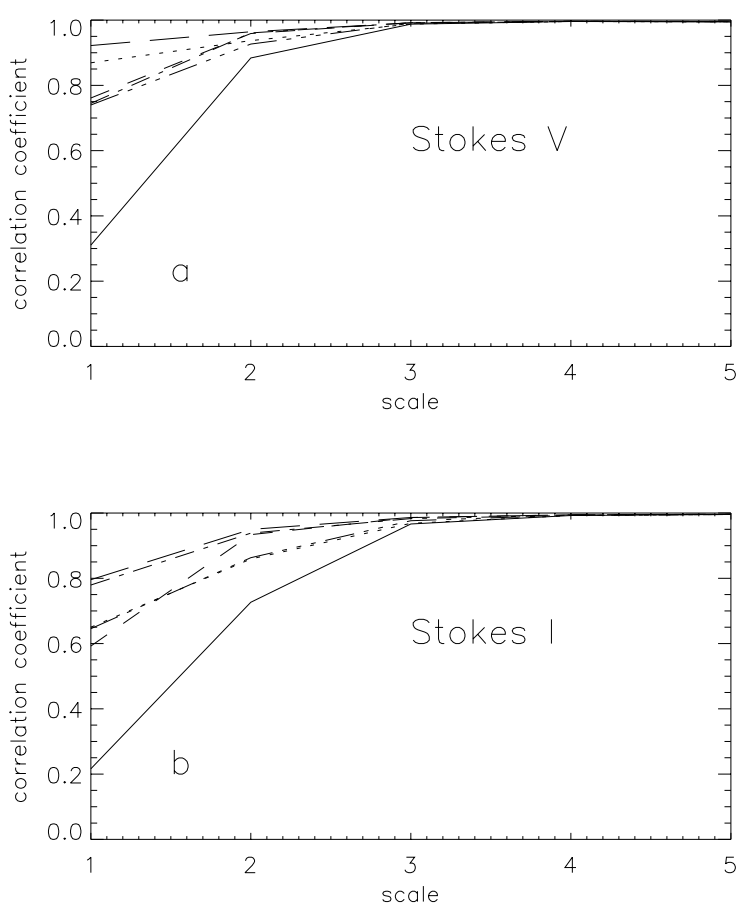

Fig. 5. Multi-resolution quality criterion of the Stokes $V$ a) and Stokes $I \mathbf{b})$ spectra. The noisy signal is represented by a solid line. Also plotted are the signal de-noised by Fourier smoothing (dotted), hierarchical thresholding (short dashed), structure detection (dash dotted), wavelet shrinkage (dash triple dotted) and wavelet-packets (long dashed), respectively. In Fig. $5 \mathrm{~b}$ the Fourier and Wavelet shrinkage curves coincides almost completely. For both spectra, the 3-level decomposition gives the closest approximation of the original noise-free signal at every resolution

scale-space plane by calculating the correlation coefficient between corresponding scales. In this manner the signals are compared at different resolutions. The results are presented in Fig. 5. The noisy signal (solid line) becomes almost identical to the original signal for scales higher than 4. This is, again, due to the fact that noise is mostly concentrated at high frequencies. It is therefore not necessary 
Table 2. Numerical evaluation of a de-noised Stokes $I$ spectrum

\begin{tabular}{lcclcl}
\hline & Fourier & $\begin{array}{c}\text { Wavelet } \\
\text { shrinkage } \\
\sigma^{2}\left[\cdot 10^{-5}\right]\end{array}$ & $\begin{array}{l}\text { Hierarchical } \\
\text { thresholding } \\
\sigma^{2}\left[\cdot 10^{-5}\right]\end{array}$ & $\begin{array}{l}\text { Structure } \\
\text { detection } \\
\sigma^{2}\left[\cdot 10^{-5}\right]\end{array}$ & $\begin{array}{l}\text { wavelet-packets } \\
\text { 3-level decomposition } \\
\sigma^{2}\left[\cdot 10^{-5}\right]\end{array}$ \\
\hline Whole spectrum & 7.4 & 6.5 & 4.9 & 5.9 & 4.4 \\
Strong lines & 11.4 & 11.4 & 9.1 & 11.0 & 7.0 \\
Weak lines & 12.2 & 9.8 & 8.9 & 7.5 & 6.9 \\
"Continuum" & 5.2 & 1.9 & 2.3 & 3.7 & 2.6 \\
\hline$\sigma_{\text {noise }} / \sigma$ & 1.72 & 1.84 & 2.10 & 1.93 & 2.23 \\
\hline
\end{tabular}

to calculate more than four scales of the wavelet transform. The wavelet-packets technique (long dashes) is again found to be superior to all of the tested methods. The additional decomposition of each scale leads to a very high fidelity between the noise-free and the de-noised spectrum at all resolution levels.

\section{Conclusions}

Provided the correct de-noising procedure is chosen, wavelet based de-noising methods give better results than classical Fourier filtering. Due to the additional spatial resolution and the frequency-adapted width of the wavelets, they are able to preserve sharp features of a signal while at the same time strongly suppressing noise within the low frequency parts. Our tests demonstrate, however, that the choice of wavelet basis and de-noising technique is crucial. We find that, at least for the considered astronomical spectra, hard thresholding using well-known orthogonal wavelet bases, such as the various Daubechies wavelets, give results that are generally not better than the best Fourier filter as far as their ability to recover the higher SNR features (spectral lines) is concerned. Donoho's wavelet shrinkage technique (based on orthogonal wavelets) and thresholding or Wiener-filtering techniques using non-orthogonal wavelets (specifically linear or cubic spline wavelets combined with the so-called $\grave{a}$ trous algorithm) as proposed by Starck \& Bijaoui (1994) work better and in general are more successful than Fourier techniques in recovering the original signal. If an efficient, quick and simple-to-use de-noising algorithm is required then we recommend the techniques developed by Starck \& Bijaoui. The technique of Bury et al. (1996) gives excellent results as well, but is not suited to de-noise large sets of measurements at once since each spectrum has to be treated separately. Their method of modelling the noise requires that the signal is divided into different parts, each of which contains only features of a similar size.

The most exact results, however, are achieved by our wavelet-packets method, namely three-level decomposition coupled with simple hard thresholding. At least for solar spectra it separates noise from the uncorrupted signal better than any other technique we have tested. In particular, it turns out to be superior to other techniques in recovering weak signals hidden in the noise. The main disadvantage of our technique is that it is slow (although not significantly slower than iterative techniques such as the adaptive filtering methods proposed by Starck \& Bijaoui 1994). We recommend it for cases in which the highest possible accuracy is desired.

In this initial investigation we have applied the multilevel decomposition technique in a rather crude manner. Other wavelet bases and truncation schemes may lead to better results. Corresponding extensions of our technique will be presented in a following paper.

Acknowledgements. We thank Benedikt Oswald for many enlightening discussions and for making his Daubechies wavelet filtering routines available to us, David Rees for drawing the work of Donoho and co-workers to our attention and the referee Dr. Bijaoui for strongly stressing the work of his group.

\section{Appendix}

For the de-noising methods presented in Sect. 3.1 the following software packages were used: The wavelet shrinkage technique of Donoho and co-workers is available through anonymous ftp from playfair.stanford.edu for the MatLab environment. The method presented by Bury et al. (1996) is also freely accessible at location holst.obs-nice.fr:/pub/wavelet.tar.gz.

Of special interest for astronomical data-processing is MIDAS, the Munich Image Data Analysis System developed at ESO. It provides a number of data analysis and de-noising routines based on different wavelet bases. In particluar, it includes the de-noising methods of Starck \& Bijaoui.

\section{References}

Anderson B.D.O., Moore J.B., 1979, Optimal Filtering. Prentice-Hall, Englewood Cliff - N.J. 
Bevington Ph., 1969, Data Reduction and Error Analysis for the Physical Sciences. McGraw-Hill Book Company, NY

Bury P., Ennode N., Petit J.-M., Bendjoya Ph., Martinez J.-P., Pinna H., Jaud J., Balladore J.-L., 1996 (preprint)

Beyclain G., Coifman R., Rokhlin V., 1993, Fast Wavelet Transform and Numerical Algorithm, Comm. Pure Appl. Math., p. 44

Brault J.W., 1985, in High Resolution in Astronomy, Benz A., Huber M., Mayor M. (eds.), 15th Advanced Course, Swiss Society of Astrophysics and Astronomy, Geneva

Brault J.W., White O.R., 1971, A\&A 13, 169

Broch, J.T., 1990, Principles of Experimental Frequency Analysis. Elsevier Applied Science, London

Cayrel de Strobel G., Spite M., 1988, The Impact of Very High S/N Spectroscopy on Stellar Physics. Kluwer, Dordrecht, IAU Symposium No. 132

Chui C.K., 1992a, An Introduction to Wavelets. Academic Press, Boston

Chui C.K., 1992b, Wavelets: A Tutorial in Theory and Applications. Academic Press, Boston

Cohen A., Daubechies I., 1992, Comm. Pure Appl. Math. 45, 1

Comtes J.M., Grossmann A., 1989, Wavelets-Time-Frequency Methods and Phase Space. Springer Verlag, Berlin

Daubechies I., 1988, Comm. Pure Appl. Math. XLI, 909

Daubechies I., 1990, IEEE Trans. Inform. Theory 36, 961

Daubechies I., 1992, Ten Lectures on Wavelets, SIAM, Philadelphia

Daubechies I., Grossmann A., Meyer Y., 1986, Math. Phys. 27, 1271

Donoho D.L., 1992a, Wavelet Shrinkage and W.V.D.: A 10Minute Tour, Technical Report, Department of Statistics, Stanford University

Donoho D.L., 1992b, De-Noising via Soft-Thresholding, Technical Report, Department of Statistics, Stanford University

Donoho D.L., Johnstone I.M., 1992, Minimax Estimation via Wavelet Shrinkage, Technical Report, Department of Statistics, Stanford University
Donoho D.L., Johnstone I.M., 1993, Ideal Spatial Adaptation via Wavelet Shrinkage, Technical Report, Department of Statistics, Stanford University

Donoho D.L., Johnstone I.M., 1994, Adapting to Unknown Smoothness via Wavelet Shrinkage, Technical Report, Department of Statistics, Stanford University

Grossmann A., Morlet J., 1984, SIAM J. Math. 15, 723

Huang T.S., 1975, Picture Processing and Digital Filtering. Springer Verlag, Berlin

Lu J., 1993, Signal Recovery and Noise Reduction with Wavelets, Ph.D. Thesis, Dartmouth College

Mallat St., 1989, IEEE Trans. Pattern Anal. Machine Intell. 11,674

Mallat St., Hwang W.L., 1992, IEEE Trans. Inform. Theory 38,617

Mallat St., Zhong S., 1992, IEEE Trans. Pattern Anal. Machine Intell. 14, 710

Meyer Y., 1993, Wavelets: Algorithms \& Applications, Society for Industrial and Applied Mathematics, Philadelphia

Murtagh F., Starck J.-L., Bijaoui A., 1995, A\&AS 112, 179

Oswald B., 1993, The Orthogonal Wavelet Transform and its Application to Astronomical Data Processing, Semesterarbeit, Institut of Astronomy, ETH, Zürich

Press H.W., Flannery B.P., Teucholsky S.A., Vetterling W.T., 1992, Numerical Recipes, 2nd edition. Cambridge University Press, Cambridge

Ruskai M.B., 1992, Wavelets and their Applications. Jones and Bartlett, Boston

Solanki S.K., 1987, The Photospheric Layers of Solar Magnetic Flux Tubes, PhD thesis No. 8309, ETH, Zürich

Starck J.-L., Bijaoui A., 1994, Signal Processing 35, 195

Starck J.-L., Murtagh F., 1994, A\&A 288, 342

Starck J.-L., Murtagh F., Bijaoui A., 1995, Astron. Data Analysis Software \& Systems IV, ASP Conf. Ser., 77

Stenflo J.O., Harvey J.W., Brault J.W., Solanki S., 1984, A\&A 131,333

Stix M., 1991, The Sun. Springer-Verlag, Berlin

Wickerhauser M.V., 1991, INRIA Lectures on Wavelet Packets Algorithms, Yale University, New Haven 